\title{
Expression of aquaporins and PAX-2 compared to CD10 and cytokeratin 7 in renal neoplasms: a tissue microarray study
}

\author{
Peter R Mazal, Martin Stichenwirth, Anke Koller, Sabine Blach, Andrea Haitel and \\ Martin Susani \\ Department of Clinical Pathology, Medical University of Vienna, AKH Vienna, Vienna, Austria
}

\begin{abstract}
Diagnostic use of antibodies against aquaporin water channel proteins and PAX-2, a nuclear transcription factor in renal development, was tested in 202 renal neoplasms, using tissue microarray technique. Immunohistochemistry for aquaporin-1, aquaporin-2, PAX-2, CD10, and cytokeratin 7 was performed on 102 clear cell renal cell carcinomas, 44 papillary renal cell carcinomas (among them 34 type 1 and 10 type 2), 24 chromophobe renal cell carcinomas, three collecting duct carcinomas (carcinomas of the collecting ducts of Bellini), and 29 oncocytomas. Aquaporin-1 expression was found in clear cell renal cell carcinomas and papillary renal cell carcinomas of both types ( 78 and $73 \%$, respectively), but not in chromophobe renal cell carcinomas, collecting duct carcinomas, and oncocytomas. Aquaporin-2 expression was not seen in any of the tested tumors. PAX-2 and CD10 was found in the majority of clear cell renal cell carcinomas (88 and $85 \%$, respectively) but only in few papillary renal cell carcinomas, chromophobe renal cell carcinomas and oncocytomas. Decrease or loss of aquaporin-1 and PAX-2 was shown in higher grades compared to lower grades of clear cell renal cell carcinomas $(P<0.0001$ and $<0.0245$, respectively). Cytokeratin 7 was rarely seen in clear cell renal cell carcinomas, type 2 papillary renal cell carcinomas, and oncocytomas, but was found in the majority of type 1 papillary renal cell carcinomas $(97.1 \%)$ and chromophobe renal cell carcinomas $(88 \%)$. Aquaporin-1 and PAX-2 expression was found to correlate with nuclear grading for clear cell renal cell carcinomas but not for papillary renal cell carcinomas. No correlation of tumor stage and aquaporin-1 and PAX2 expression was seen. Aquaporin-1 and PAX-2 are reliable markers for clear cell renal cell carcinomas of lower grades but not for higher grades. CD10 expression remains stable, independent of nuclear grading.

Modern Pathology (2005) 18, 535-540, advance online publication, 22 October 2004; doi:10.1038/modpathol.3800320
\end{abstract}

Keywords: aquaporin-1; PAX-2; renal cell carcinoma; renal neoplasms; tissue multiarray

We tested the potential diagnostic use of antibodies against aquaporins, water channel proteins found in different segments of the renal tubulary system, ${ }^{1-3}$ and PAX-2 a member of the paired box family and nuclear transcription factor in development of renal epithelia. ${ }^{4}$ The expression of these antigens was analyzed in different types of renal tumors. Aquaporin-1, aquaporin-2 and PAX-2 were compared to two established antibodies, CD10 and cytokeratin 7, which are in use in renal tumor pathology. Decrease or loss of these antigens depending on tumor grading and pT staging was assessed, to get information on the diagnostic reliability of different

Correspondence: Dr M Susani, MD, Department of Clinical Pathology, Medical University of Vienna, AKH Vienna, Waehringer Guertel 18-20, A-1090 Vienna, Austria.

E-mail: martin.susani@meduniwien.ac.at

Received 1 April 2004; revised 17 September 2004; accepted 20 September 2004; published online 22 October 2004 markers, depending on tumor differentiation. Renal neoplasms comprise several kinds of different tumors. In most cases it is possible to distinguish different types of renal neoplasms on the basis of conventionally stained tissue sections, alone. However, overlapping morphological characteristics can sometimes pose difficulties to make a proper diagnosis. Diagnostic problems can eventually arise in discriminating tumors like some chromophobe renal cell carcinomas, oncocytomas, and the granular variant of clear cell renal cell carcinoma. ${ }^{5-7}$ As different types of renal neoplasms have different prognosis, many immunohistochemical markers have been tested to support a correct histological diagnostic decision. In this study, we examined 202 renal neoplasms, including renal cell carcinomas, as well as oncocytomas, using immunohistochemistry and tissue microarray technology which is a useful tool for efficient and rapid analysis of protein expression by immunohistochemistry. ${ }^{8-10}$ 


\section{Materials and methods}

\section{Patients}

The study included 202 paraffin-embedded tumors from the archives of the Department of Clinical Pathology, Medical University of Vienna. Specimens were all sliced before they were routinely fixed overnight with $4.5 \%$ buffered formaldehyde. The group of renal cell carcinomas included exclusively primary tumors: 102 of clear cell, 44 of papillary (34 type 1 and 10 type 2), 24 of chromophobe, and three of collecting duct type (carcinoma of the collecting ducts of Bellini). Benign renal tumors included 29 oncocytomas. All tumors were re-evaluated. Carcinomas were classified according to the recommendations of the WHO Classification 2004, ${ }^{11}$ staged according to the TNM-System, ${ }^{12}$ and graded according to Fuhrman et al. ${ }^{13}$ Papillary carcinomas were separated in type 1 and type 2, according to Delahunt and Eble. ${ }^{14}$ None of the patients had been treated with chemotherapy prior to surgery. Pathological grading and staging of renal carcinomas is summarized in Table 1.

\section{Construction of Tissue Microarrays}

Hematoxylin- and eosin-stained slides of all tumors were reviewed and representative areas were selected. These were areas with characteristic histomorphology of the individual tumor, including the highest grade. In Grade 4 tumors areas with spindle-shaped cells were seen in different amounts between 5 and $75 \%$. True sarcoma areas were not found. A total of 606 tumor tissue cylinders with a diameter of $0.6 \mathrm{~mm}$ were punched out from those regions of the corresponding paraffin blocks. From each tumor, three tissue cylinders were taken. Additionally, nine punches of regular tissues, including renal cortex, renal medullary tissue, and pelvic urothelium from tumor-free renal parenchyma were collected. All tissue cylinders were precisely arrayed in receptor paraffin blocks, using a tissue microarrayer system (Beecham ${ }^{\circledR}$, CA, USA). The last six positions at the right corner of the tissue microarrays were left free to enable correct orientation of the sections. In addition to immunohistochemical evaluation, histology of all sections was reviewed, to ensure that the sample of deeper portions of the tumor cores remained representative.

\section{Immunohistochemistry}

Sections, 4- $\mu \mathrm{m}$ thick, were cut from the tissue microarrays and transferred to silanized glass slides. Sections were stained with hematoxylin and eosin and with antibodies listed in Table 2. Signal detection was performed with the avidin-biotin reaction, using peroxidase, except in cytokeratin 7 staining, where alkaline phosphatase was used. Antigen retrieval for cytokeratin 7 was performed by microwave pretreatment in citrate buffer ( $\mathrm{pH}$ 6.0) for $10 \mathrm{~min}$ with $450 \mathrm{~W}$, and for CD10 by autoclave pretreatment for $10 \mathrm{~min}$ with $450 \mathrm{~W}$. For aquaporin1, aquaporin-2, and PAX-2 this was done by microwave pretreatment for $20 \mathrm{~min}$ at $120 \mathrm{~W}$, and $3 \times 5 \mathrm{~min}$ each at $450 \mathrm{~W} .^{15}$

\section{Scoring and Statistical Analysis}

Sections were scored using a semiquantitative scale for each individual tumor tissue cylinder on the array slide, with - for negative staining, + for focal staining $(<10 \%$ of cells showing immunoreac tivity), ++ for staining of $10-50 \%$ of tumor cells, +++ for diffuse staining $(>50 \%$ of cells showing immunoreactivity), and 0 for uninterpretable

Table 2 Antibodies

\begin{tabular}{llll}
\hline Antibody & Source & Dilution & Antigen retrieval \\
\hline Aquaporin-1 & Chemicon & $1: 400$ & MW \\
Aquaporin-2 & Chemicon & $1: 50$ & MW \\
PAX-2 & Zymed & $1: 100$ & MW \\
CD10 & Novocastra & $1: 40$ & AC \\
Cytokeratin 7 & Dako & $1: 200$ & MW
\end{tabular}

MW, microwave pretreatment; AC, autoclave pretreatment.

Table 1 Grading and staging of renal cell carcinomas

\begin{tabular}{|c|c|c|c|c|c|c|c|c|c|c|c|c|}
\hline & \multicolumn{4}{|c|}{ CCRCC $(\mathrm{n}=102)$} & \multicolumn{4}{|c|}{$P R C C(\mathrm{n}=44)$} & \multicolumn{2}{|c|}{ ChrRCC $(\mathrm{n}=24)$} & \multicolumn{2}{|c|}{$C D C(\mathrm{n}=3)$} \\
\hline & G1 & G2 & G3 & G4 & G1 & $G 2$ & G3 & G4 & G2 & G3 & G3 & G4 \\
\hline pT1a & 3 & 22 & 8 & - & 1 & 10 & 3 & 1 & 11 & - & - & - \\
\hline pT1b & - & 9 & 8 & - & - & 8 & - & - & 3 & 1 & - & - \\
\hline pT2 & 3 & 3 & 1 & 2 & - & 1 & - & 1 & 1 & 2 & - & - \\
\hline pТ3а & 2 & 16 & 6 & 6 & - & 9 & 6 & 1 & 2 & 3 & 2 & 1 \\
\hline pT3b & 1 & 4 & 6 & 2 & - & 2 & 1 & - & 1 & - & - & - \\
\hline
\end{tabular}

CCRCC, clear cell renal cell carcinoma; PRCC, papillary renal cell carcinoma; ChrRCC, chromophobe renal cell carcinoma; CDC, carcinoma of the collecting ducts of Bellini.

No pT3c and pT4 tumors in our series. No G1 and G4 tumors in ChrRCC group, no G1 and G2 tumors in CDC group. 
(excessive background staining, loss of representative tissue, not enough tumor tissue, etc.). Scores were entered into a Microsoft Excel spreadsheet. Uninterpretable results (0-results) were not used for statistical calculations. Score results for triplicate tissue cylinders were collected as median results. Kruskal-Wallis test and post-hoc Tukey test were used to analyze and compare immunohistochemical scoring results, tumor type, grading and staging in carcinomas. Calculations were done using SAS, Version 8.1 software. Correction for making multiple comparisons was made, and a $P$-value of $<0.05$ was considered statistically significant.

\section{Results}

\section{Immunohistochemical Results in Normal Renal Parenchyma}

In the nine tissue cores with normal renal parenchyma included in the microarrays, strong membranous aquaporin-1 staining was seen in the epithelia of all proximal renal tubules, in the descending limb of Henle, in glomerular endothelial cells, and extraglomerular capillaries. Aquaporin-2 was detected in the epithelia of collecting ducts. PAX-2 expression could not be detected in normal renal parenchyma. CD10 antigen was shown in the epithelia of all proximal tubules and in glomerular epithelial cells. Cytokeratin 7 was expressed in the epithelia of distal tubules, collecting ducts, and urothelial cells of the renal pelvis.

\section{Immunohistochemical Results in Renal Neoplasms}

\section{Aquaporin-1 scoring results}

Aquaporin-1 expression was seen in the majority of all renal cell carcinomas, but only in one single case $(3.5 \%)$ of renal oncocytomas. This tumor with characteristic oncocytic histology demonstrated prominent diffuse membranous staining. All other oncocytomas were completely negative. In the carcinoma group, clear cell renal cell carcinomas and papillary renal cell carcinomas demonstrated membranous expression of aquaporin-1 in 78 and $73 \%$, respectively. No significant difference was found comparing type 1 and type 2 papillary renal cell carcinomas. Chromophobe renal cell carcinomas and collecting duct carcinomas were negative.

\section{Aquaporin-2 scoring results}

Aquaporin-2 was not detected in any of the examined renal neoplasms.

\section{PAX-2 scoring results}

Nuclear staining of PAX-2 transcription factor was seen in the majority of renal cell carcinomas, and in $14 \%$ of renal oncocytomas, including the case of oncocytoma with aquaporin-1 expression. In the carcinoma group, clear cell renal cell carcinomas demonstrated expression of PAX-2 in $88 \%$. It was also detected in $18 \%$ of papillary renal cell carcinomas, $13 \%$ of chromophobe renal cell carcinomas, and not in collecting duct carcinomas. No significant difference was found comparing type 1 and type 2 papillary renal cell carcinomas.

\section{CD10 scoring results}

Expression of CD10 antigen was seen in the majority of renal cell carcinomas, but only in one case $(3.5 \%)$ of renal oncocytomas. This was the same oncocytoma which showed also aquaporin-1 and PAX-2 expression. In the carcinoma group, clear cell renal cell carcinomas demonstrated expression of CD10 antigen in $85 \%$, papillary renal cell carcinomas in $23 \%$, and chromophobe renal cell carcinomas in only $4 \%$. Collecting duct carcinomas were negative.

Cytokeratin 7 scoring results

Cytokeratin 7 was seen in $39 \%$ of all renal cell carcinomas, and in $7 \%$ of renal oncocytomas. In the carcinoma group, clear cell renal cell carcinomas demonstrated expression of cytokeratin 7 in only $8 \%$, but in $77 \%$ of all papillary renal cell carcinomas (97\% type $1,30 \%$ type 2 ), $88 \%$ of chromophobe renal cell carcinomas, and in one of three collecting duct carcinomas. Table 3 and Figure 1 summarize the immunohistochemical results.

\section{Influence of Tumor Grading on Expression Profile in Renal Cell Carcinomas}

Comparing immunohistochemical results with grading within specific subtypes of carcinomas, significant differences were seen in clear cell renal cell carcinomas, with decrease or loss of aquaporin-1 and PAX-2 correlating with increasing nuclear grades $(P<0.0001$ and $<0.0245$, respectively). For papillary renal cell carcinomas, a decrease of aquaporin-1 was noted with increasing grading, however, this difference was not statistically significant. CD10 expression did not show correlation with tumor grading.

\section{Influence of Tumor Staging (pT) on Expression Profile in Renal Cell Carcinomas}

Comparing immunohistochemical results of carcinomas with different pT stages within the specific tumor groups, we did not find significant correlations with the studied markers.

\section{Discussion}

Aquaporins are water-transporting proteins, expressed in many epithelial tissues and endothelium ${ }^{3}$ and in some urinary tract neoplasms. ${ }^{15-17}$ In the normal kidney, aquaporin-1 is only expressed in epithelial cells of proximal tubules and in the 
Table 3 Immunohistochemical results in renal cell carcinomas and oncocytomas (\% positive cases)

\begin{tabular}{|c|c|c|c|c|c|c|}
\hline & & CCRCC & PRCC & ChrRCC & $C D C$ & Oncocytoma \\
\hline \multirow[t]{4}{*}{ Aquaporin-1 (AQP-1) } & Total & 78 & 73 & 0 & 0 & 3.5 \\
\hline & + & 2 & 2 & 0 & 0 & 0 \\
\hline & ++ & 27 & 7 & 0 & 0 & 0 \\
\hline & +++ & 49 & 64 & 0 & 0 & 3.5 \\
\hline Aquaporin-2 (AQP-2) & Total & 0 & 0 & 0 & 0 & 0 \\
\hline \multirow[t]{4}{*}{ PAX-2 } & Total & 88 & 18 & 13 & 0 & 14 \\
\hline & + & 6 & 4.5 & 4 & 0 & 3.5 \\
\hline & ++ & 23 & 9 & 9 & 0 & 7 \\
\hline & +++ & 59 & 4.5 & 0 & 0 & 3.5 \\
\hline \multirow[t]{4}{*}{ CD10 } & Total & 85 & 23 & 4 & 0 & 3.5 \\
\hline & + & 3 & 4.5 & 0 & 0 & 0 \\
\hline & ++ & 20 & 14 & 0 & 0 & 0 \\
\hline & +++ & 62 & 4.5 & 4 & 0 & 3.5 \\
\hline \multirow[t]{4}{*}{ Cytokeratin-7 (CK7) } & Total & 8 & 77 & 88 & 33 & 7 \\
\hline & + & 3 & 0 & 4 & 0 & 3.5 \\
\hline & ++ & 0 & 13 & 0 & 33 & 3.5 \\
\hline & +++ & 5 & 64 & 84 & 0 & 0 \\
\hline
\end{tabular}

CCRCC, clear cell renal cell carcinoma; PRCC, papillary renal cell carcinoma; ChrRCC, chromophobe renal cell carcinoma; CDC, carcinoma of the collecting ducts of Bellini.
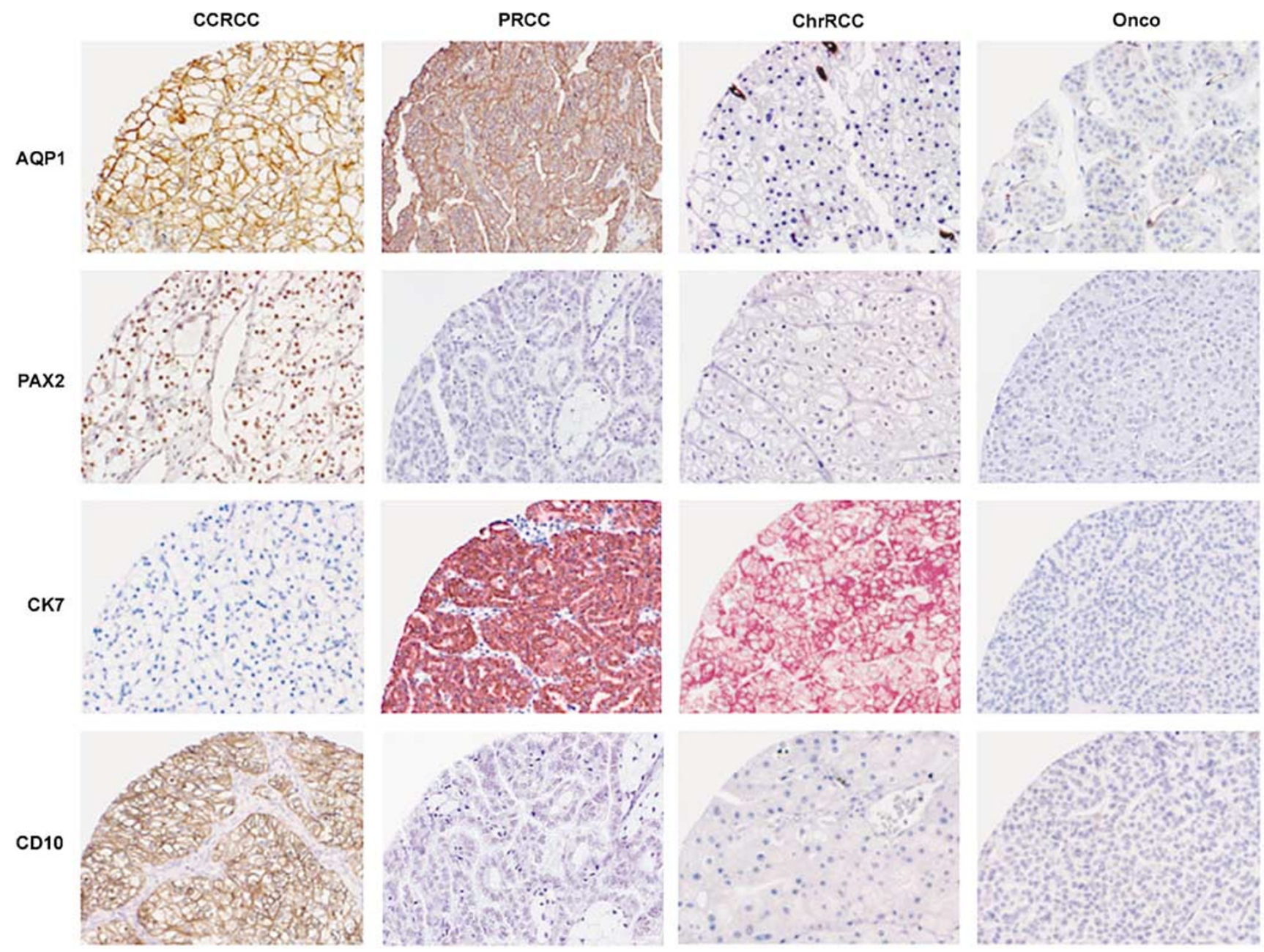

Figure 1 Typical expression patterns of different antigens in renal cell cancers and oncocytomas. CCRCC, clear cell renal cell carcinoma: PRCC, papillary renal cell carcinoma; ChrRCC, chromophobe renal cell carcinoma; AQP1, aquaporin-1; CK7, cytokeratin 7. 
descending limb of Henle, but not in other nephron segments, collecting ducts or urothelial cells, whereas aquaporin-2 is selectively expressed in collecting ducts. ${ }^{1,2}$ Only two studies of aquaporin-1 and aquaporin-2 in small series of renal cell carcinomas have been published in the literature. ${ }^{16,17}$ However, these studies are based primarily on Northern blot analysis and in situ hybridization, and no classification of renal carcinomas with subtypes was done. Therefore, we tested the diagnostic utility of aquaporin-1 and -2 on paraffin-embedded tissue of common types of renal cell carcinomas and renal oncocytomas. We found prominent expression of aquaporin-1 in clear cell renal cell carcinomas and papillary renal cell carcinomas, but not in chromophobe renal cell carcinomas or in collecting duct carcinomas. These results support the widely accepted histogenetic concept of the origin of clear cell renal cell carcinoma and papillary renal cell carcinoma from the proximal part of the nephron, and the origin of chromophobe carcinoma and renal oncocytoma from intercalated cells in collecting tubules. ${ }^{18}$

Aquaporin-1 expression decreased significantly in higher grades of clear cell renal cell carcinoma, compared to lower grades of this tumor group. This was seen in clear cell renal cell carcinomas but not in papillary renal cell carcinomas. Oncocytomas were usually negative. Aquaporin-2 was not expressed in our series, including three collecting duct carcinomas.

PAX-2 belongs to a family of transcription factors. It is required for development and proliferation of renal tubules. PAX-2 expression is downregulated in mature tissue, and usually no expression is seen in the healthy, adult kidney. ${ }^{4}$ In one recent study, 56 renal tumors were analyzed by frozentissue immunohistochemistry. ${ }^{19}$ In this study, Daniel et al demonstrated expression of PAX-2 in more than $90 \%$ of 33 clear cell renal cell carcinomas and in $100 \%$ of 12 papillary renal cell carcinomas. They also demonstrated some reactivity in chromophobe renal cell carcinomas and oncocytomas. Our study is the first, testing PAX-2 expression in a large number of paraffinembedded renal tumors. Our results are similar, demonstrating PAX-2 expression in $88 \%$ of clear cell renal cell carcinomas, and reactivity in some chromophobe renal cell carcinomas and oncocytomas. However, expression of PAX-2 in papillary renal cell carcinomas was significantly lower with $18 \%$ of papillary renal cell carcinomas in our series, compared to $100 \%$ in Daniels frozen sections. We have no explanation for this discrepancy, but emphasize on the fact that comparing immunohistochemical results from frozen tissue with those from paraffin tissue may be difficult with some antibodies.

Regarding correlation of PAX-2 and tumor grading, we observed a significant decrease of PAX-2 expression in higher grades of clear cell renal cell carcinomas compared to lower grades. This was seen in clear cell renal cell carcinomas, but not in papillary renal cell carcinomas.

Our results regarding CD10 and cytokeratin 7 are in accordance with those of $\mathrm{Kim}$ and $\mathrm{Kim},{ }^{6}$ and Avery et $a l,{ }^{20}$ reporting, that the majority of CD10positive renal tumors are clear cell renal cell carcinomas and papillary renal cell carcinomas. Kim and $\mathrm{Kim}^{6}$ and Delahunt and Eble $^{14}$ demonstrated that type-1 papillary renal cell carcinomas and chromophobe renal cell carcinomas express cytokeratin 7, whereas clear cell renal cell carcinomas and oncocytomas are usually negative, which was also found in our study. Cytokeratin 7 can therefore be a useful marker for the differentiation of chromophobe renal cell carcinomas and oncocytomas. Regarding correlation of CD10 and cytokeratin 7 , and tumor grading, no significant differences were observed.

In conclusion, we found significant expression of aquaporin-1 in clear cell renal cell carcinomas and papillary renal cell carcinomas but typically not in chromophobe renal cell carcinomas and in oncocytomas. Aquaporin-2 was not detected in renal carcinomas and oncocytomas. PAX-2 and CD-10 expression was found in the majority of clear cell renal cell carcinomas. No significant correlation of pT stage with aquaporin-1 or PAX-2 expression could be demonstrated. Clear cell renal cell carcinomas with higher grades showed a significant decrease or loss of aquaporin-1 and PAX-2 compared to lower grades in clear cell renal cell carcinomas. Higher grades in papillary renal cell carcinomas did not.

Aquaporin-1 and PAX-2 are reliable markers for clear cell renal cell carcinomas of lower grades but not for higher grades, whereas CD10 expression remains stable with no significant differences comparing nuclear grades.

\section{References}

1 Agre P, Nielsen S. The aquaporin family of water channels in kidney. Nephrology 1996;17:409-415.

2 Nielsen S, Agre P. The aquaporin family of water channels in kidney. Kidney Int 1995;48:1057-1068.

3 Verkman AS. Physiological importance of aquaporin water channels. Ann Med 2002;34:192-200.

4 Dahl E, Koseki H, Balling R. Pax genes and organogenesis. Bioessays 1997;9:755-765.

5 Khoury JD, Abrahams NA, Levin HS, et al. The utility of epithelial membrane antigen and vimentin in the diagnosis of chromophobe renal cell carcinoma. Ann Diagn Pathol 2002;6:154-158.

$6 \mathrm{Kim}$ MK, Kim S. Immunohistochemical profile of common epithelial neoplasms arising in the kidney. Appl Immunohistochem Mol Morphol 2002; 10:332-338.

7 Koller A, Kain R, Haitel A, et al. Renal oncocytoma with prominent intracytoplasmic vacuoles of mitochondrial origin. Histopathology 2000;37:264-268. 
8 Kononen J, Bubendorf L, Kallioniemi A, et al. Tissue microarray for high-throughput molecular profiling of tumor specimens. Nat Med 1998;4:844-847.

9 Moch H, Schraml P, Bubendorf L, et al. Highthroughput tissue microarray analysis to evaluate genes uncovered by cDNA microarray screening in renal cell carcinoma. Am J Pathol 1999;154: 981-986.

10 Zhang DH, Salto-Tellez M, Putti TC, et al. Reliability of tissue microarrays in detecting protein expression and gene amplification in breast cancer. Mod Pathol 2003;16:79-85.

11 Eble JN, Sauter G, Epstein JI, et al (eds). World Health Organization Classification of Tumours. Tumours of the Urinary System and Male Genital Organs. IARC Press: Lyon, 2004, pp 9-87.

12 Sobin LH. Kidney In: Sobin LH, Wittekind CH (eds). International Union Against Cancer. TNM Classification of Malignant Tumors, 6th edn. Wiley: New York, 2002, pp 180-183.

13 Fuhrman SA, Lasky LC, Simas C. Prognostic significance of morphologic parameters in renal cell carcinoma. Am J Surg Pathol 1982;6:655-663.
14 Delahunt B, Eble JN. Papillary renal cell carcinoma: a clinicopathologic and immunohistochemical study of 105 tumors. Mod Pathol 1997;10:537-544.

15 Mazal PR, Schaufler R, Altenhuber-Müller R, et al. Derivation of nephrogenic adenomas from renal tubular cells in kidney transplant recipients. N Engl J Med 2002;347:653-659.

16 Kageyama Y, Sasaki S, Yamamura Y, et al. Water channel protein subtype suggests the origin of renal cell carcinoma. J Urol 1996;156:291-295.

17 Takenawa J, Kaneko Y, Kishishita M, et al. Transcript levels of aquaporin 1 and carbonic anhydrase IV as predictive indicators for prognosis of renal cell carcinoma patients after nephrectomy. Int J Cancer 1998;79:1-7.

18 Störkel S, Steart PV, Drenchkahn D, et al. The human chromophobe renal cell carcinoma: its probable relation to intercalated cells of the collecting duct. Virchows Arch B Cell Pathol 1989;56:237-245.

19 Daniel L, Lechevallier E, Giorgi R, et al. Pax-2 expression in adult renal tumors. Hum Pathol 2001;32:282-287.

20 Avery AK, Beckstead J, Renshaw AA, et al. Use of antibodies to RCC and CD10 in the differential diagnosis of renal neoplasms. Am J Surg Pathol 2000;24:203-210. 